\title{
Changes in Fine Structure of Prolamellar Body in Relation to the Formation of the Chloroplast*
}

\author{
by Taiji IKEDA**
}

Received December 15, 1969

\begin{abstract}
The formation of the prolamellar body was studied using the seedlings of Cucumis sativus in relation to the development of lamellar structure in the plastids. The seedlings were grown in the light for various lengths of time, and then transferred to darkness. The prolamellar bodies were formed in a plastid in the dark, and increased in number as the lamellar structure developed. The formation of prolamellar bodies in the plastid, in which the grana lamellae differentiate, seems to occur only in the region of intergrana lamellae. Besides, the present experiment showed that the prolamellar bodies in continuous dark are devoid of lamellar structure, and that they are also produced when the seedling is transferred to the darkness at its earlier stage of development. Moreover, the prolamellar body and lamellar structure are formed simultaneously under the weak light condition, while the former disappears under stronger light. From these observations, it is suggested that the appearance of prolamellar body may be caused by blocking the conversion of vesicles into lamellae.
\end{abstract}

Since the electron microscopic observations were made by Heitz and Leyon $(1954)^{1,2)}$ on the aggregation of vesicles at an initial stage of chloroplast development, a number of investigations have been reported concerning the fine structure of the vesicle aggregate. Such a vesicular structure has been called in various terms such as "dense core" by Leyon (1954)2), "Heitz-Leyonsche Krystalle" by Menke $(1962,1963)^{3,4)}$, "prolamellar body" by Hodge $(1956)^{5}$, and so on. The vesicle aggregates of a crystalline structure have been recognized in the dark grown plastids of Phaseolus (G. Eriksson 19616), H. I. Virgin et al. 19637), S. Klein et al. $1964^{8)}$ ), Avena (B. E. S. Gunning 1967) ${ }^{9}$, barley and its genetic mutants (von Wettstein 1958)(10), Elodea (Mühlethaler und Frey-Wyssling 1959) ${ }^{11}$, and Arabidopsis thariana $\left(J\right.$. Velemínský 1966) ${ }^{12}$. According to K. Mühlethaler and Frey-Wyssling (1959) ${ }^{11}$, the formation of a prolamellar body occurs, as a rule, in etiolated plants, but not in plants grown under sufficient light. Gunning $(1967)^{9}$, R. G. Everson $(1967)^{13)}$, and M. Wrischer $(1966)^{14}$ have also made similar observations that the vesicles of a crystalline prolamellar body are gradually dissociated and the double lamellae come into appearance in the light. As a rule, one or two prolamellar bodies were observed in these plastids. On the other hand, according to von Wettstein $(1958)^{10)}$, about 100 prolamellar bodies were formed in Picea cotyledone which was cultivated in the dark at Kobe.

* This work was presented at the Annual Meeting of the Bot. Soc. Japan in Oct. 1967

** Department of Biology, Faculty of Science, Niigata University, Niigata, Japan. 
following the initial illumination. In the study of M. Wrischer $(1966)^{14)}$, many crystalline prolamellar bodies were also formed under dim light condition (below 300 lux) and were called "weak light prolamellar bodies'..

A similar situation was also observed by the present study in the plastids cultured under the condition of light-dark alteration. The present paper deals with these changes in number of prolamellar bodies during the formation of plastids and discusses possible relationship between the occurrence of prolamellar bodies and the developmental stage of plastids.

\section{Materials and Methods}

It has been shown that the development of plastids in the cotyledon of Phaseolus vulgaris begins with the growth of a primary leaf. In this experiment, therefore, the process of greening was taken as a measure using the cotyledons of Cucumis sativus. Seeds of this plant were soaked with water and allowed to germinate at $20^{\circ}$ in the light ( $\left.1300 \mathrm{lux}\right)$. The seeds under beginning germination were divided into several groups, each being illuminated for $12,24,36,48,63,72$ hours and then placed in the dark for 24 hours. The seeds grown in continuous darkness were used as control. In parallel, the seeds were grown under 300 lux, in order to examine the effect of dim light upon the formation of a prolamellar body. Then, the seedlings were cut into small pieces (ca. $1 \mathrm{~mm}^{2}$ ) and fixed with $2.5 \%$ solution of glutaraldehyde in $0.1 \mathrm{M}$-phosphate buffer $(\mathrm{pH} 7.4)$ for 4 hours. The post-fixation was made with $1.25 \%$ solution of $\mathrm{KMnO}_{4}$ in the same buffer for 2 hours. Fixed materials were throughly washed with distilled water, dehydrated with a series of acetone, embedded in an epoxy-resin according to $\mathrm{Luft}^{15}$, and finally polymerized by heating at $60^{\circ}$. Sections were made by cutting with a JUM$5 \mathrm{~A}$ ultra-microtome, and stained with uranyl acetate at $50^{\circ}$ for 2 hours. A JEMT7 electron microscope was used for the observations.

\section{Results}

The plastid in cotyledon cultured in the dark for 72 hours usually contains only one prolamellar body, in which a radial protrusion of several poor primary layers may be observed (Fig. 1). The prolamellar body exhibits a characteristic honeycomb-like arrangement of tubules, as has been reported by previous workers. In a seedling grown in the dark for 24 hours following the initial 39 hour-illumination, the plastids were $2 \mu$ in diameter and had only one prolamellar body with several protrusions of incomplete primary layers (Fig. 3). This is quite similar to the case of seedlings grown under continuous darkness for 72 hours, where no differentiated grana was observed. In the seedlings cultured in the dark after short illumination such as 12 and 24 hours, the morphology of plastids showed no difference from that of the seedlings grown under continuous darkness. However, the dark treatment following illumination of more than 48 hours was effective for the formation of two or three prolamellar bodies within the well developed lamellar system, in which primary layers were arranged in parallel in the same direction and 2-3 stacks of grana lamellae were clearly differentiated (Fig. 4). In these plastids, a number of incomplete lamellae were developed as protrusions from each prolamellar body. The dark treatment following illumination longer than 63 hours 
was favorable for the development of a lamellar system together with the formation of several stacks of grana lamellae; the size of a prolamellar body being decreased in spite of the increase in its number (Fig. 5). The prolamellar bodies that appeared in the plastids fully etiolated by the dark treatment for 87 hours were ca. $1.3 \mu$ in diameter (Fig. 2), while those grown under the condition of 63 hour-light and subsequent 24 hour-dark were only $0.5 \mu$ on an average. However, the appearance of their substructure was quite similar in both cases.

The plastids kept in the dark after the initial illumination for 72 hours grew up to $6 \mu$ in diameter and possessed the stack made up of more than ten grana lamellae and many small prolamellar bodies, e. g., 9-10 bodies as seen in Fig. 6. The development of lamellar system in the plastids however, seemed to be remarkably suppressed and remained in the developmental stage at the transferring of the seedling from the light to the dark. Moreover, it should be noted that the prolamellar bodies usually appeared at the sites of intergrana lamellae but not in the stroma of plastids. In these plastids, infolding of vesicles from inner membrane of the plastid was not observed.

In order to clarify whether or not the prolamellar body is formed even in a fully matured plastid, the materials preliminarily illuminated under 1300 lux for 17 days were kept in the dark. The result showed that the prolamellar body is not produced in the mature plastid, but only in the growing plastid. The mode of interconversion between prolamellar bodies and lamellar structures may be shown by the observation that the smaller prolamellar bodies appeared at a greening stage easily disappear under 1300 lux illumination only for 20 minutes, while the prolamellar bodies in etiolated seedlings do not disappear even after 8 hour-illumination.

Under the weak light condition, the prolamellar body appeared in the plastid at an initial stage of greening, and remained practically unchanged even at a later stage of plastid development, where the grana stacks with 7-8 lamellae had already

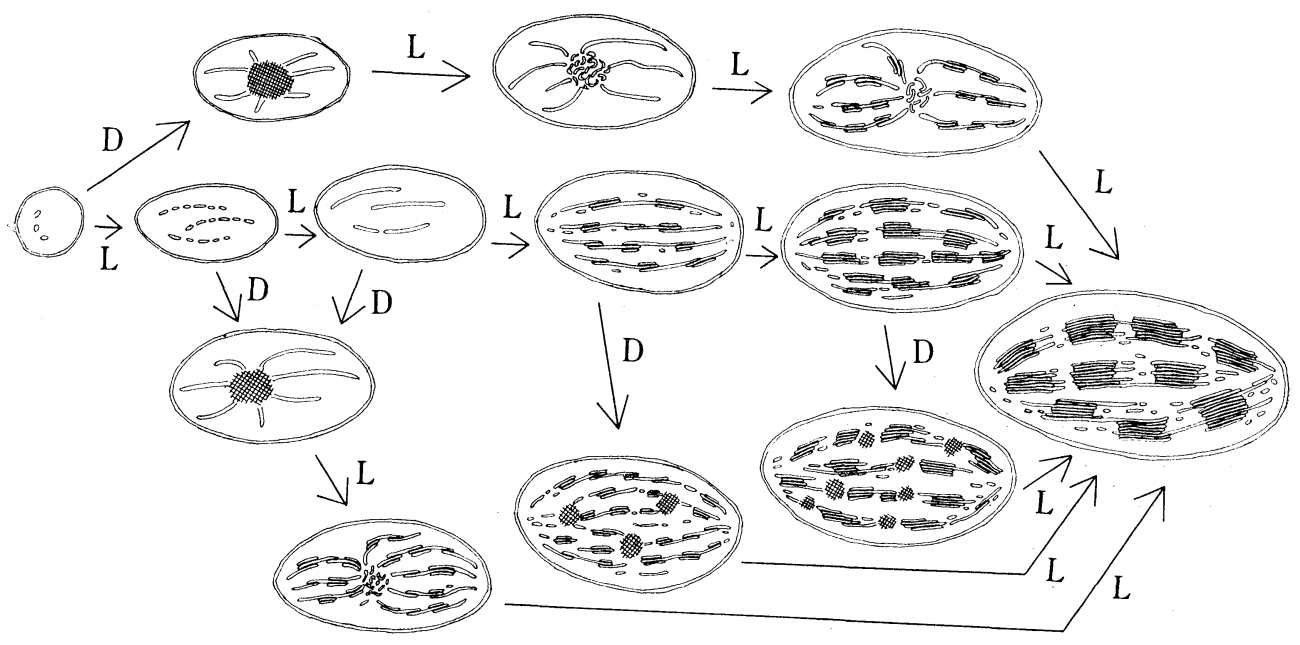

Fig. 7. Possible scheme showing the formation and subsequent change of prolamellar body in the plastid. $\mathrm{D}$ : process in the dark $\mathrm{L}$ : process in the light 
appeared. In this case, only one prolamellar body was formed at an earlier stage, and it gradually increased in number as the plastid developed. The same situation was observed for the materials grown in the dark after initial illumination as described above.

In Fig. 7, the formation of prolamellar bodies is summarized diagrammatically in relation to the development of plastid.

\section{Discussion}

Several investigations have been carried out on the mode of occurrence of a prolamellar body in the plastid in relation to the light and some other factors. Von Wettstein $(1958)^{10)}$ as well as Mühlethaler and Frey-Wyssling (1959) ${ }^{11)}$ reported that the development of lamellar system in plants grown in the light can take place without the formation of a prolamellar body. In a recent decade, Eilam and Klein $(1962)^{16)}$ showed that in bean seedlings the prolamellar bodies formed in the dark disappear by the illumination of $400 \mathrm{ft}$-c. for 24 hours and then normal lamellar formation takes place. In these seedlings, one or two prolamellar bodies were formed in a single plastid, since the dark culture was made at an initial stage of growth. M. Wrischer $(1966)^{14)}$ observed a number of "weak light prolamellar bodies" in the plastid of Phaseolus vulgaris cultured under the weak light condition. However, the relationship between these two kinds of prolamellar bodies has not been sufficiently elucidated until now.

In this experiment, therefore, an attempt was made to investigate this problem transferring the plant materials from a strong light condition to darkness. The result showed that the appearance of prolamellar bodies varies with various stages of plastid development; for instance, the number of prolamellar bodies in a plastid increases nearly in parallel with the development of plastids. It seems quite likely that the nature of the "weak light prolamellar bodies" is identical with that of the prolamellar bodies observed in etiolated plants. Under the weak light condition such as 300 lux, prolamellar bodies were formed in the plastid when it was at an early stage of development, and they remained almost unchanged until now. Upon strong illumination, however, prolamellar bodies were not formed and the "weak light prolamellar bodies" that had been produced immediately disappeared.

Furthermore, Eriksson $(1961)^{6)}$ showed that in bean seedlings the prolamellar body is replaced by a lamellar system under strong illumination for about 3 hours $\left(\mathrm{S}\right.$. Klein 1964) ${ }^{8)}$. The present study showed that the conversion of vesicles, namely the structural unit of a prolamellar body, into a lamellar structure occurs more readily in "weak light prolamellar bodies" than in etiolated plastids.

On the other hand, Hodge $(1956)^{5)}$ had an opinion that a prolamellar body may be formed in a plastid when the synthesis of vesicles predominates over their conversion into lamellae. In the meantime, Klein $(1960)^{17)}$ reported that the grana- and stroma-lamellae must be formed by rearrangement of the vesicles constituting a prolamellar body. In addition, Klein $(1961)^{18)}$ and Boardman $(1962)^{192}$ have suggested the presence of some precursors of chlorophyll in the prolamellar bodies in etiolated plastid. In this connection, it was shown in the present study that small prolamellar bodies are formed mainly at the sites of intergrana lamellae.

Now, the results of the present work may be summarized as follows. (1) As a rule, prolamellar bodies are formed in the dark, while the production of vesicles occurs independently of light. (2) When the plant grown in the light is transferred 
to dark condition, the prolamellar bodies are formed in the plastids at any stage of development. (3) Prolamellar bodies are formed in parallel with the development of lamellae under the weak light condition, and they disappear under stronger illumination.

From these facts, it is concluded that the light energy is needed for the conversion of vesicles into lamellae, but not for the synthesis of vesicles. Accordingly, the appearance of prolamellar body may take place by blocking the conversion of vesicles into lamellae during its synthesis. But still, it is not clear what mechanism does participate in the conversion of vesicles into the characteristic lattice structure of the prolamellar bodies.

The present author wishes to express his sincere thanks to Prof. K. Hayashi and Dr. S. Tôyama in the Botanical Institute, Tokyo Kyoiku University, and also to Assist. Prof. Y. Yoshida in the Department of Biology, Faculty of Science, Niigata University, for their deliberate guidance and helpful suggestions throughout this experiment.

This work was supported by the grant from the Ministry of Education, for which thanks are also given by the author.

\section{References}

1) Heitz, E., Expt1. Cell Res. $7: 606$ (1954).

2) Leyon, H., Exptl. Cell Res. $7: 609$ (1954).

3) Menke, W., Z. Naturforschg. 17b: 188 (1962).

4) Menke, W., Z. Naturforschg. 18b: 821 (1963).

5) Hodge, A. J., et al., J. Biophys. Biochem. Cytol. 2 : 597 (1956).

6) Eriksson G., et al., Ber. Deutsch. bot. Ges. $74: 221$ (1961).

7) Virgin, H. I., et al., Photochem. Photobiol. 2 : 83 (1963).

8) Klein, S., et al., J. Cell Biol. 22: 443 (1964).

9) Gunning, B.E.S., and Jagoe, M.P., Biochemistry of Chloroplasts Vol. 2: 655 (1967).

10) von Wettstein, D., Brookhaven Symposia in Biology No. 11: 138 (1958).
11) Mühlethaler, K., und Frey-Wyssling, A., J. Biophys. Biochem. Cytol. 6 : 507 (1959).

12) Velemínský, J., und Röbbelen, G., Planta $68: 15$ (1966).

13) Everson, R. G., and Gibbs, M., Plant Physiol. 42 : 1153 (1967).

14) Wrischer, M., Z. Pflanzenphysiol. 55 : 269 (1966).

15) Luft, J.H., J. Biophys. Biochem. Cytol. 9: 409 (1961).

16) Eilam, Y., and Klein, S., J. Cell Biol. 14 : 169 (1962).

17) Klein, S., J. Biophys. Biochem. Cytol. 8 : 529 (1960).

18) Klein, S., et al., J. Biophys. Biochem. Cytol. 11 : 433 (1961).

19) Boardman, N.K., et al., Biochim. Biophys. Acta $59: 222$ (1962). 


\section{Explanation of the Figures}

Fig. 1. The plastid grown in the dark for 72 hours, one prolamellar body being observed in a plastid. $\times 18400$

Fig. 2. The plastid grown in the dark for 96 hours, the prolamellar body becoming larger and more distinct than the above. $\times 20000$

Fig. 3. The plastid in the seedling grown in the dark for 24 hours subsequent to initial 39 hour-illumination, only one prolamellar body being observed in a plastid. $\times 19200$

Fig. 4. The plastid in the seedling grown in the dark for 24 hours after initial illumination for 48 hours. Grana lamellae have developed, and three prolamellar bodies are observed in a plastid. $\times 18400$

Fig. 5. The plastid in the seedling grown in the dark for 24 hours after initial illumination for 63 hours. Grana lamellae have much more developed, and six prolamellar bodies can be seen in a plastid. $\times 18400$

Fig. 6. The plastid in the seedling grown in the dark 24 hours following initial illumination for 72 hours. Many prolamellar bodies are formed in a plastid, each being located in the region of intergrana lamellae. $\times 19200$ 

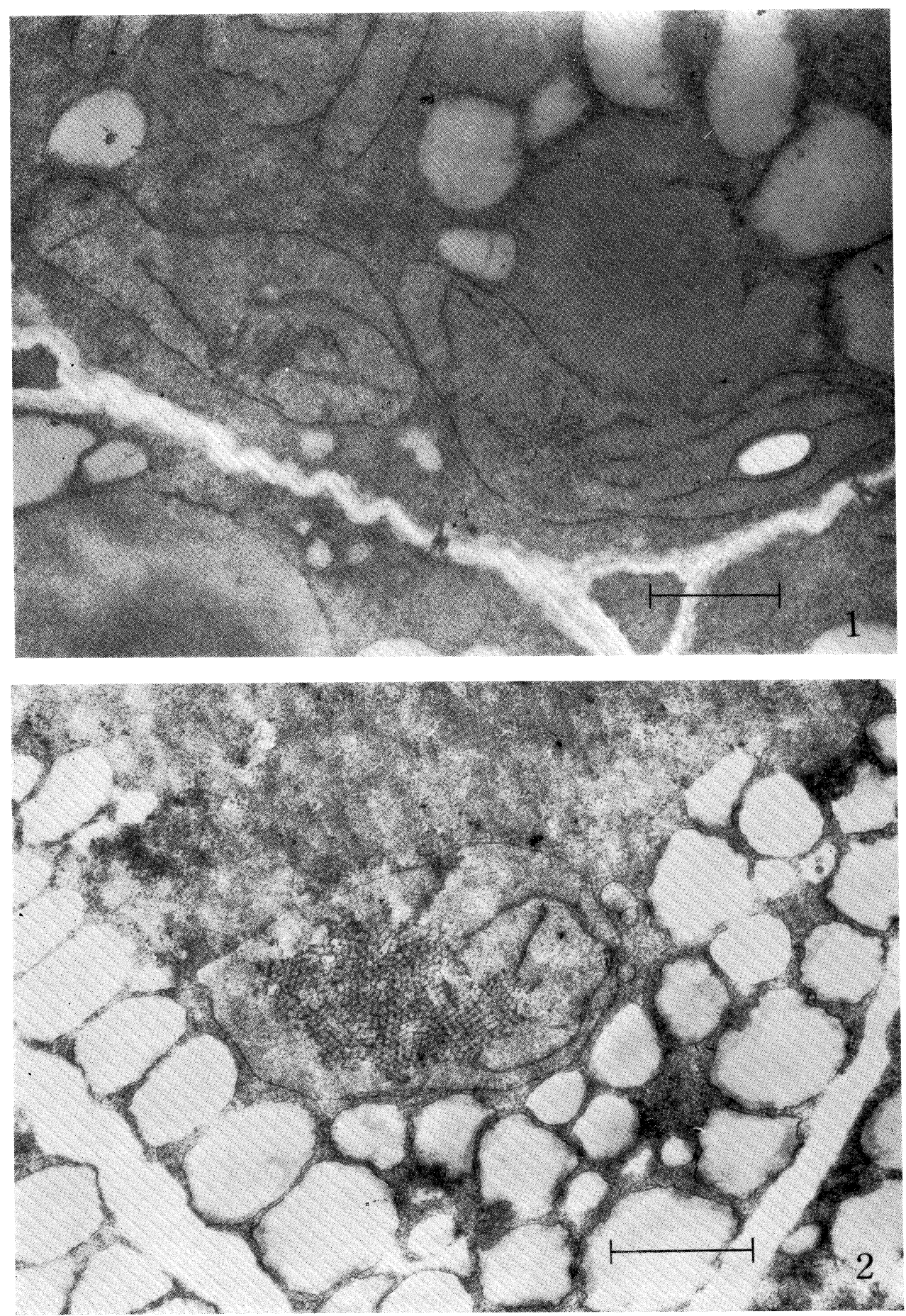

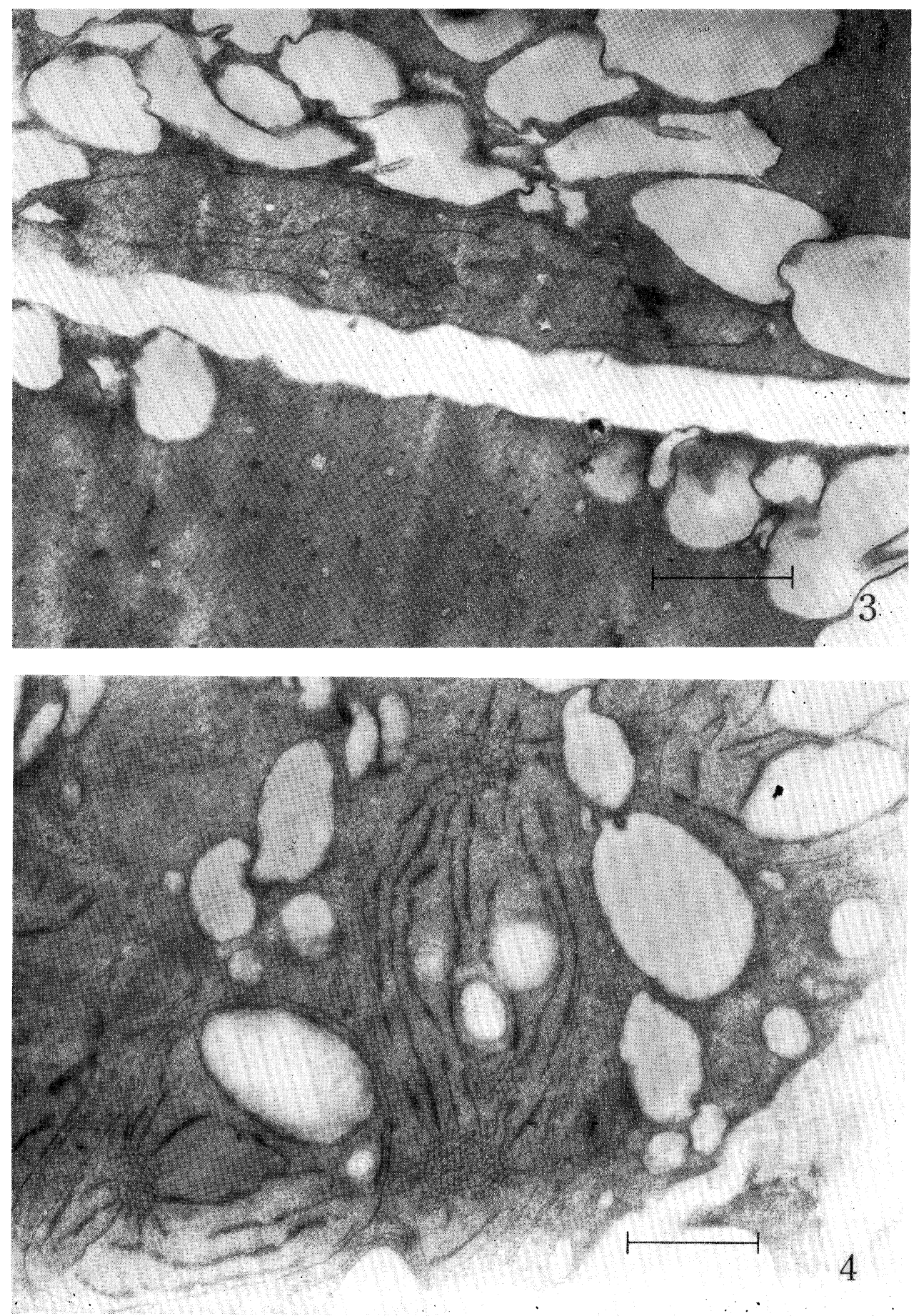

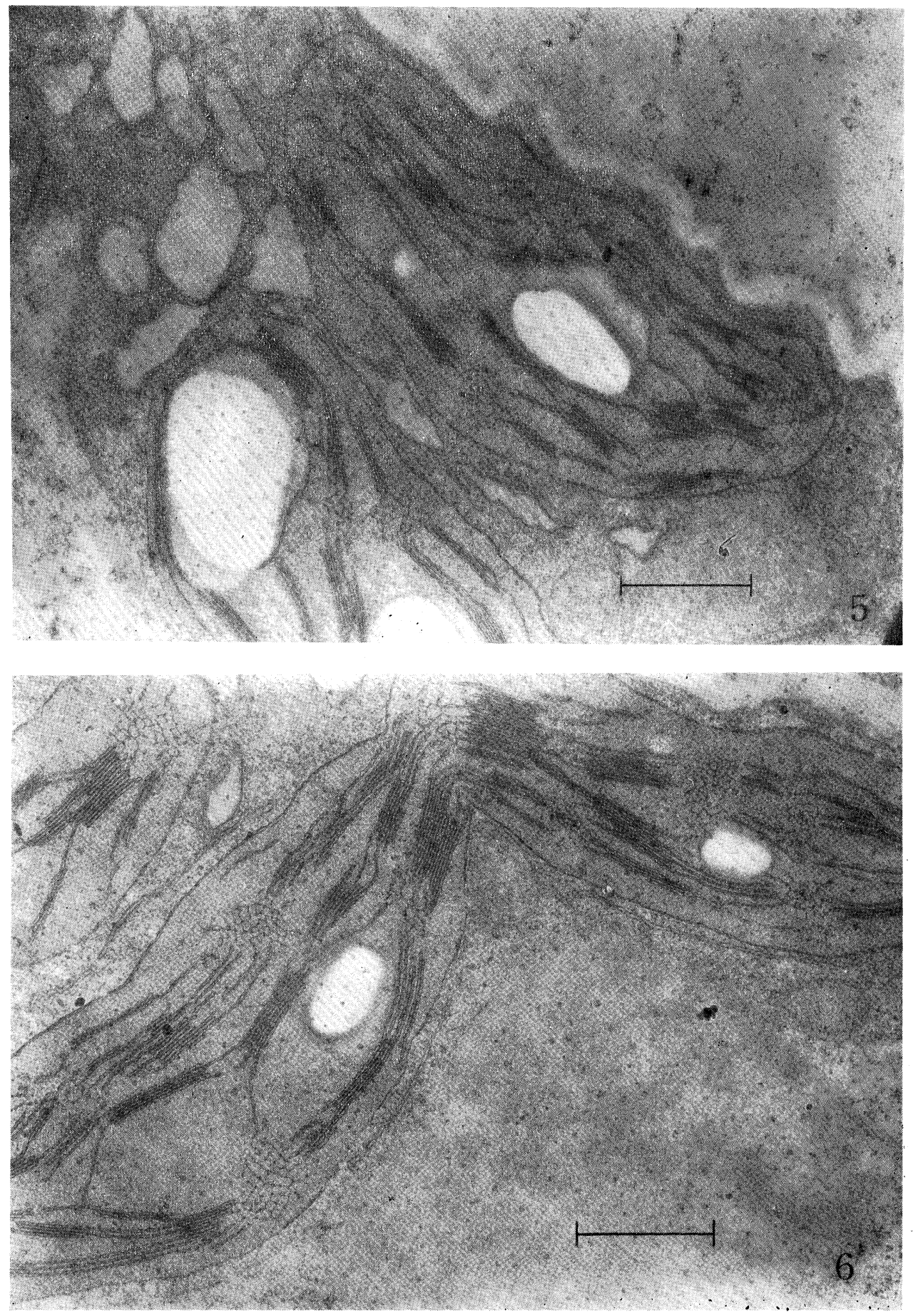